\title{
Odd Perfect Numbers Not Divisible by 3 Are Divisible by at Least Ten Distinct Primes
}

\author{
By Masao Kishore
}

\begin{abstract}
Hagis and McDaniel have shown that the largest prime factor of an odd perfect number $N$ is at least 100111 , and Pomerance has shown that the second largest prime factor is at least 139 . Using these facts together with the method we develop, we show that if $3 \nmid N, N$ is divisible by at least ten distinct primes.
\end{abstract}

1. Introduction. A positive integer $N$ is called perfect if $\sigma(N)=2 N, \sigma(N)$ being the sum of positive divisors of $N$. No odd perfect (OP) numbers are known; however, it has been proved that if $N$ is OP and $\omega(N)$ denotes the number of distinct prime factors of $N$, then $\omega(N) \geqslant 5$ by Sylvester (1888), Dickson (1913) and Kanold (1949); $\omega(N) \geqslant 6$ by Gradstein (1925), Kühnel (1949) and Weber (1951); $\omega(N) \geqslant 7$ by Pomerance (1972, [1]) and Robbins (1972); $\omega(N) \geqslant 8$ by Hagis (1975, [3]); and that if $N$ is OP and $3 \nmid N$, then $\omega(N) \geqslant 8$ by Sylvester $(1888)$, and $\omega(N) \geqslant 9$ by Kanold (1949, [6] ). Also, it has been proved that if $N$ is OP, then $N>10^{200}$ by Buxton and Elmore (1976, [5]), the largest prime factor of $N>100110$ by Hagis and McDaniel (1975, [4]), and the second largest prime factor of $N \geqslant 139$ by Pomerance (1975, [2]).

In this paper we prove

THEOREM. If $N$ is $O P$ and $3 \nmid N, \omega(N) \geqslant 10$.

2. Preliminary results. Throughout this paper let

$$
N=\prod_{i=1}^{r} p_{i}^{a_{i}}
$$

where $p_{1}<p_{2}<\cdots<p_{r}$ are odd primes and $a_{1}, \ldots, a_{r}$ are positive integers. We call $p_{i}^{a_{i}}$ a component of $N$ and write $V_{p_{i}}(N)$ for $a_{i}$.

Euler proved that if $N$ is OP, then for some $j, p_{j} \equiv a_{j} \equiv 1$ (4) and for $i \neq j$, $a_{i} \equiv 0$ (2). $p_{j}$ is called the special prime denoted by $\Pi$.

Lemma 1. Suppose $N$ is $O P, 3 \nmid N$, and $p^{a}$ is a component of $N$. If $p \equiv 2$ (3), then $p \neq \Pi$, and if $p \equiv 1$ (3), then $a \neq 1$ (3).

Proof. If $p \equiv 2$ (3) and $p=\Pi$, then $\sigma\left(p^{a}\right) \equiv 0$ (3) because $a$ is odd, while if $p$ $\equiv 1$ (3) and $a \equiv 2(3)$, then $\sigma\left(p^{a}\right) \equiv 0(3)$, both of which contradict the fact that $3 \nmid$ N. Q.E.D.

From Euler's Theorem and Lemma 1 we have

Corollary 1. Suppose $N$ is $O P, 3 \nmid N$, and $p^{a}$ is a component of $N$. If $p \equiv$ 1 (4) and $p \equiv 1$ (3), then $a=1,4,6,9,10,12, \ldots$; if $p \equiv 1$ (4) and $p \equiv 2$ (3), 
then $a=2,4,6,8,10,12, \ldots$; if $p \neq \equiv 1$ (4) and $p \equiv 1$ (3), then $a=4,6,10,12$, $\ldots$; if $p \neq \equiv 1$ (4) and $p \equiv 2$ (3), then $a=2,4,6,8,10,12, \ldots$.

We are interested in finding $p_{i}^{a_{i}}$, s for which

$$
\prod_{i=1}^{r} S\left(p_{i}^{a_{i}}\right)=2, \quad \text { where } S\left(p^{a}\right)=\sigma\left(p^{a}\right) / p^{a} .
$$

Since the accuracy of the computer is limited, we use the inequality

$$
0.693147180<\log 2=\sum_{i=1}^{r} \log S\left(p_{i}^{a_{i}}\right)<0.693147181 .
$$

With nine-digit figures we have sufficient accuracy, and with log we can easily control computational errors involved.

Suppose $N$ is OP, $3 \nmid N$, and $p^{a}$ is a component of $N$. We define

$a(p)=\operatorname{minimum}\{b \mid b>1$ is an allowable power of $p$ as determined by Corollary 1 and $\left.p^{b+1}>10^{9}\right\}$

and

$$
L\left(p^{a}\right)= \begin{cases}{\left[10^{9} \log S\left(p^{a}\right)\right] 10^{-9}} & \text { if } a<a(p), \\ {\left[10^{9} \log \frac{p}{p-1}\right] 10^{-9}} & \text { if } a \geqslant a(p),\end{cases}
$$

where [ ] is the greatest integer function.

We note that if $p$ and $q$ are odd primes with $p<q$, then for any positive integers $a$ and $b$

$$
S\left(q^{a}\right)<\frac{q}{q-1}<\frac{p+1}{p} \leqslant S\left(p^{b}\right)
$$

and so $L\left(q^{a}\right) \leqslant L\left(p^{b}\right)$.

LEMMA 2. Suppose

$$
N=\prod_{i=1}^{r} p_{i}^{a_{i}}
$$

is $O P$ and $3 \nmid N$. Then

$$
S_{r}<\sum_{i=1}^{r} L\left(p_{i}^{b_{i}}\right)<T_{r},
$$

where $S_{r}=0.693147180-r 10^{-9}, T_{r}=0.693147181+r 10^{-9}, b_{i}=a_{i}$ if $a_{i}<$ $a\left(p_{i}\right)$, and $b_{i}=a\left(p_{i}\right)$ if $a_{i} \geqslant a\left(p_{i}\right)$.

Proof. Since $N$ is OP, (1) holds. Suppose $p^{a}$ is a component of $N$. If $a<a(p)$, then

$$
\left|\log S\left(p^{a}\right)-L\left(p^{a}\right)\right|<10^{-9}
$$

If $a \geqslant a(p)$, then

$$
\begin{aligned}
10^{-9} & \geqslant \log \frac{p}{p-1}-L\left(p^{a}\right)>\log S\left(p^{a}\right)-L\left(p^{a(p)}\right) \\
& \geqslant \log \frac{p^{a+1}-1}{p^{a+1}-p^{a}}-\log \frac{p}{p-1}=\log \left(1-\frac{1}{p^{a+1}}\right)=-\sum_{i=1}^{\infty} \frac{1}{i\left(p^{a+1}\right)^{i}} \\
& >-\sum_{i=1}^{\infty} \frac{1}{\left(p^{a+1}\right)^{i}}=\frac{-1}{p^{a+1}-1} \geqslant-10^{-9}
\end{aligned}
$$


and so

$$
\left|\log S\left(p^{a}\right)-L\left(p^{a(p)}\right)\right|<10^{-9}
$$

Hence,

$$
\left|\sum_{i=1}^{r} \log S\left(p_{i}^{a_{i}}\right)-\sum_{i=1}^{r} L\left(p_{i}^{b_{i}}\right)\right|<r 10^{-9},
$$

and (2) follows from (1) and (3). Q.E.D.

We also need the following lemmas, which were proved in [1, pp. 269-271]:

LEMMA 3. If $q$ is a prime for which $q-1$ is a power of $2, N$ is $O P$, and if $p^{a}$ is a component of $N$, then

$$
V_{q}\left(\sigma\left(p^{a}\right)\right)= \begin{cases}V_{q}(a+1) & \text { if } p \equiv 1(q) \\ V_{q}(p+1)+V_{q}(a+1) & \text { if } p \equiv-1(q) \text { and } p=\Pi \\ 0 & \text { otherwise. }\end{cases}
$$

LEMMA 4. If $N$ is $O P, p^{a}$ is a component of $N$, and if $q$ is a prime and $q^{b} \mid a+1$, then $N$ is divisible by at least $b$ distinct primes $\equiv 1(q)$ other than $p$.

LEMMA 5. If $n$ is $O P, 17^{a}$ is a component of $N$, and if $17^{a}+\Pi+1$, then $N$ is divisible by at least two primes $\equiv 1$ (17).

3. Proof of the Theorem. In this section, we shall prove that if $3 \nmid N$ and $\omega(N)=9$, then $N$ is not OP.

LEMMA 6. If $N$ is $O P, 3 \nmid N$, and if $\omega(N)=9$, then

$$
\begin{gathered}
p_{1}=5, \quad p_{2}=7, \quad p_{3}=11, \quad p_{4}=13, p_{5} \leqslant 19, \quad p_{6} \leqslant 23, \quad p_{7} \leqslant 53, \\
p_{8} \geqslant 139 \text { and } p_{9}>100110 .
\end{gathered}
$$

Proof. By [4] $p_{9}>100110$, and by [2] $p_{8} \geqslant 139$. Others follow from

$$
\begin{aligned}
& \frac{5}{4} \frac{7}{6} \frac{11}{10} \frac{17}{16} \frac{19}{18} \frac{23}{22} \frac{29}{28} \frac{139}{138} \frac{100111}{100110}<2, \\
& \frac{5}{4} \frac{7}{6} \frac{11}{10} \frac{13}{12} \frac{23}{22} \frac{29}{28} \frac{31}{30} \frac{139}{138} \frac{100111}{100110}<2, \\
& \frac{5}{4} \frac{7}{6} \frac{11}{10} \frac{13}{12} \frac{17}{16} \frac{29}{28} \frac{31}{30} \frac{139}{138} \frac{100111}{100110}<2,
\end{aligned}
$$

and

$$
\frac{5}{4} \frac{7}{6} \frac{11}{10} \frac{13}{12} \frac{17}{16} \frac{19}{18} \frac{59}{58} \frac{139}{138} \frac{100111}{100110}<2 \text {. Q.E.D. }
$$

LEMMA 7. $p_{5}=17$ in Lemma 6.

Proof. Suppose $p_{5}=19$. Then $p_{6}=23, p_{7}=29$ and $p_{8}=139$ because

$$
\frac{5}{4} \frac{7}{6} \frac{11}{10} \frac{13}{12} \frac{19}{18} \frac{23}{22} \frac{31}{30} \frac{139}{138} \frac{100111}{100110}<2
$$

and 


\section{$\frac{5}{4} \frac{7}{6} \frac{11}{10} \frac{13}{12} \frac{19}{18} \frac{23}{22} \frac{29}{28} \frac{149}{148} \frac{100111}{100110}<2$.}

Hence

$$
N=5^{a} 17^{a}{ }_{1} 1^{a} 3_{13^{a}} 19^{a} 523^{a} 69^{a} 7139^{a} p_{9}^{a 9} .
$$

Since

$$
\frac{5}{4} \frac{7}{6} S\left(11^{2}\right) \frac{13}{12} \frac{19}{18} \frac{23}{22} \frac{29}{28} \frac{139}{138} \frac{100111}{100110}<2
$$

and

$$
\frac{5}{4} \frac{7}{6} \frac{11}{10} S\left(13^{1}\right) \frac{19}{18} \frac{23}{22} \frac{29}{28} \frac{139}{138} \frac{100111}{100110}<2,
$$

$a_{3} \neq 2$ and $a_{4} \neq 1$. Also, $a_{2}, a_{4}, a_{5}, a_{8} \neq 2$ by Corollary 1 . Since every odd prime factor of $\sigma\left(p_{i}^{a_{i}}\right)$ is a factor of $N, a_{1}, a_{6}, a_{7} \neq 2$ and $a_{1}, a_{2} \neq 4$ because $31 \mid \sigma\left(5^{2}\right)$, $71\left|\sigma\left(5^{4}\right), 2801\right| \sigma\left(7^{4}\right), 79 \mid \sigma\left(23^{2}\right)$ and $67 \mid \sigma\left(29^{2}\right)$. Hence for $1 \leqslant i \leqslant 2, a_{i} \geqslant 6$ and for $3 \leqslant i \leqslant 8, a_{i} \geqslant 4$. Then $N$ is not OP because

$$
S(N)>\prod_{i=1}^{8} S\left(p_{i}^{a_{i}}\right)>2 \text {. Q.E.D. }
$$

LEMMA 8. $17^{a} \mid \Pi+1$ and $\Pi>100110$ in Lemma 6 .

Proof. Suppose $17^{a} \nmid \Pi+1$. Since $p_{i} \not \equiv \pm 1$ (17) for $1 \leqslant i \leqslant 7, p_{8} \equiv p_{9} \equiv$ 1 (17) by Lemma 5 . If $17^{2} \mid \sigma\left(p_{j}^{a_{j}}\right)$ for $j=8$ or 9 , then by Lemma $3,17^{2} \mid a_{j}+1$, and by Lemma $4 N$ would be divisible by at least two primes $\equiv 1$ (17) other than $p_{j}$. Hence $17^{2} \nmid \sigma\left(p_{j}^{a}{ }_{j}\right)$. Since $17 \nmid \sigma\left(p_{i}^{a_{i}}\right)$ for $1 \leqslant i \leqslant 7$, we conclude that $a_{5}=2$, $17 \mid \sigma\left(p_{8}^{a_{8}}\right)$ and $17 \mid \sigma\left(p_{9}^{a_{9}}\right)$. Then $p_{8}=\sigma\left(17^{2}\right)=307$, and for $j=8,9, a_{j}=16, p_{j} \neq$ П, $5 \nmid \sigma\left(p_{j}^{a_{j}}\right)$, and so for some $1 \leqslant i \leqslant 7,5 \mid \sigma\left(p_{i}^{a_{i}}\right)$. By Lemma 3 and Corollary $1, p_{i}$ $=11,31$, or 41 , and $\sigma\left(p_{i}^{4}\right) \mid \sigma\left(p_{i}^{a_{i}}\right)$ because $5 \mid a_{i}+1$; however, $3221\left|\sigma\left(11^{4}\right), 17351\right|$ $\sigma\left(31^{4}\right), 579281 \mid \sigma\left(41^{4}\right)$, and none of these primes $\equiv 1(17)$. Hence $p_{i} \neq 11,31,41$, a contradiction, and $17^{a_{5}} \mid \Pi+1$.

If $a_{5} \geqslant 4, \Pi \geqslant 2 \cdot 17^{4}-1=167041$, while if $a_{5}=2, \Pi=p_{9}>100110$ because $p_{8}=307$. Q.E.D.

LEMMA 9. If $3 \nmid N, \omega(N)=9$, and if $p_{8}>1000, N$ is not $O P$.

Proof. Suppose $N$ is OP. Then by Lemma 2

$$
S_{9}<\sum_{i=1}^{9} L\left(p_{i}^{b_{i}}\right)<T_{9} .
$$

If $a_{i}<a\left(p_{i}\right), b_{i}=a_{i}$, and so every prime factor of $\sigma\left(p_{i}^{b_{i}}\right)$ is a factor of $N$ except when $p_{i}=\Pi$. Hence if

we have

$$
M=\left(\prod_{i=1}^{7} p_{i}\right)\left(\prod_{i=1 ; b_{i}<a\left(p_{i}\right)}^{7} \sigma\left(p_{i}^{b_{i}}\right)\right)
$$

$$
\begin{aligned}
& \omega(M)=7, \\
& \omega(M)=8, \quad \text { or } \\
& \omega(M)=9 .
\end{aligned}
$$


Suppose (4) holds. Since $p_{8}>1000$ and $p_{9}>100110$,

$$
S_{9}<\sum_{i=1}^{7} L\left(p_{i}^{b_{i}}\right)+\log \frac{1009}{1008}+\log \frac{100111}{100110} \text { and } \sum_{i=1}^{7} L\left(p_{i}^{b_{i}}\right)<T_{7}
$$

Suppose (5) holds, and let $p$ be the prime factor of $M$ other than $p_{i}, 1 \leqslant i \leqslant 7$. Then

$$
\begin{aligned}
& \text { if } 1000<p<100110, S_{9}<\sum_{i=1}^{7} L\left(p_{i}^{b_{i}}\right)+L\left(p^{b}\right)+\log \frac{100111}{100110} \\
& \text { if } p>100110, S_{9}<\sum_{i=1}^{7} L\left(p_{i}^{b_{i}}\right)+\log \frac{1009}{1008}+L\left(p^{b}\right), \text { and } \\
& \sum_{i=1}^{7} L\left(p_{i}^{b_{i}}\right)+L\left(p^{b}\right)<T_{8}
\end{aligned}
$$

where $b \leqslant a(p)$ is an allowable power of $p$. Suppose (6) holds. Then the two prime factors of $M$ other than $p_{i}, 1 \leqslant i \leqslant 7$, are $p_{8}$ and $p_{9}$, and

$$
p_{8}>1000, p_{9}>100110 \text { and } S_{9}<\sum_{i=1}^{9} L\left(p_{i}^{b_{i}}\right)<T_{9} .
$$

Computer was used to find $\Pi_{i=1}^{7} p_{i}^{b_{i}}$ satisfying
(A) (4) and (7),
(B) (5) and (8), or
(C) (6) and (9),

with the following results:

$$
\begin{array}{lc}
5^{12} 7^{10} 11^{8} 13^{9} 17^{8} 23^{6} 29^{6}, & 5^{12} 7^{10} 11^{8} 13^{9} 17^{8} 23^{6} 29^{4}, \\
5^{12} 7^{10} 11^{8} 13^{9} 17^{8} 23^{4} 29^{6}, & 5^{12} 7^{10} 11^{8} 13^{9} 17^{6} 23^{6} 29^{6} \\
5^{12} 7^{10} 11^{8} 13^{6} 17^{8} 23^{6} 29^{6}, & \text { or } \quad 5^{10} 7^{10} 11^{8} 13^{9} 17^{8} 23^{6} 29^{6} .
\end{array}
$$

In every case $p_{8} \geqslant 3011$ because

$$
S\left(5^{10} 7^{10} 11^{8} 13^{6} 17^{6} 23^{4} 29^{4} 3001^{1}\right)>2 .
$$

Then $N$ is not OP because $p_{9} \geqslant \Pi>17^{6}-1$ and

$$
S(N)<\frac{5}{4} \frac{7}{6} \frac{11}{10} \frac{13}{12} \frac{17}{16} \frac{23}{22} \frac{29}{28} \frac{3011}{3010} \frac{p_{9}}{p_{9}-1}<2 \text {. Q.E.D. }
$$

LEMMA 10. If $3 \nmid N, \omega(N)=9$, and if $p_{8}<1000, N$ is not $O P$.

Proof. Suppose $N$ is OP. Then by Lemma 2

$$
S_{9}<\sum_{i=1}^{9} L\left(p_{i}^{b_{i}}\right)<T_{9} \text {. }
$$

If

$$
M=\left(\sum_{i=1}^{8} p_{i}\right)\left(\prod_{i=1 ; b_{i}<a\left(p_{i}\right)}^{7} \sigma\left(p_{i}^{b_{i}}\right)\right)
$$

then 


$$
\begin{aligned}
& \omega(M)=8, \quad \text { or } \\
& \omega(M)=9 .
\end{aligned}
$$

Suppose (10) holds. Then

$$
p_{8}<1000, \quad S_{9}<\sum_{i=1}^{8} L\left(p_{i}^{b_{i}}\right)+\log \frac{100111}{100110}, \quad \text { and }
$$

$$
\sum_{i=1}^{8} L\left(p_{i}^{b_{i}}\right)<T_{8}
$$

Suppose (11) holds. Then the prime factor of $M$ other than $p_{i}, 1 \leqslant i \leqslant 8$, is $p_{9}$, and

$$
p_{8}<1000, \quad p_{9}>100110 \text { and } S_{9}<\sum_{i=1}^{9} L\left(p_{i}^{b_{i}}\right)<T_{9} .
$$

Computer was used to find $\Pi_{i=1}^{8} p_{i}^{b_{i}}$ satisfying

(A) (10) and (12), or

(B) (11) and (13),

with the following results:

$$
\begin{aligned}
& 5^{12} 7^{10} 11^{8} 13^{9} 17^{8} 19^{6} 47^{6} 233^{4}, \quad 5^{12} 7^{10} 11^{8} 13^{9} 17^{8} 19^{6} 47^{6} 233^{2}, \\
& 5^{12} 7^{10} 11^{2} 13^{9} 17^{8} 19^{6} 43^{6} 331^{4}, \quad 5^{2} 7^{10} 11^{8} 13^{9} 17^{8} 19^{6} 31^{6} 953^{4} \text {, } \\
& 5^{2} 7^{10} 11^{2} 13^{9} 17^{8} 19^{6} 31^{6} 557^{4} \text {, or } 5^{2} 7^{10} 11^{2} 13^{9} 17^{8} 19^{6} 31^{6} 557^{2} \text {. }
\end{aligned}
$$

Then $N$ is not OP because in every case $p_{9}=\Pi \geqslant 2 \cdot 17^{8}-1$ and $S(N)<2$. Q.E.D.

Lemmas 9 and 10 prove our theorem.

Computer (PDP 11 at the University of Toledo) program run time for Lemmas 9 and 10 was three minutes.

I would like to thank Professor J. Chidambaraswamy for his help in preparing this paper. Also, I would like to thank the referee who suggested the use of [2], which cut down the computer time.

\footnotetext{
Department of Mathematics

University of Toledo

Toledo, Ohio 43606
}

1. C. POMERANCE, "Odd perfect numbers are divisible by at least seven distinct primes," Acta Arith., v. 25, 1973/74, pp. 265-300. MR 49 \#4925.

2. C. POMERANCE, "The second largest prime factor of an odd perfect number," Math. Comp., v. 29, 1975, pp. 914-921. MR 51 \#8018.

3. P. HAGIS, JR., "Every odd perfect number has at least eight prime factors," Notices Amer. Math. Soc., v. 22, 1975, p. A-60. Abstract \#720-10-14.

4. P. HAGIS, JR. \& W. L. MCDANIEL, "On the largest prime divisor of an odd perfect number. II," Math. Comp., v. 29. 1975, pp. 922-924. MR 51 \#8021.

5. M. BUXTON \& S. ELMORE, “An extension of lower bounds for odd perfect numbers," Notices Amer. Math. Soc., v. 23, 1976, p. A-55. Abstract \#731-10-40.

6. H.-J. KANOLD, "Folgerungen aus dem Vorkommen einer Gauss'schen Primzahl in der Primfaktorenzerlegung einer ungeraden Vollkommenen Zahl," J. Reine Angew. Math., v. 186, 1944, pp. 25-29. MR 6, 255. 